\title{
The Evaluation of Lung Function in Very Low Birth Weight Premature Infants during Preschool Age
}

Ayse Sevim Gokalp*, Ayla Gunlemez and Seda Uyan

Department of Pediatrics, Division of Neonatology and Chest, University of Kocaeli, Turkey

*Corresponding author: Ayse Sevim Gokalp, Professor of Pediatrics, Division of Neonatology, Kocaeli University, 41380, Kocaeli, Turkey, E- mail: gokalpas@kocaeli.edu.tr

Received date: Jan 08, 2015; Accepted date: Jan 09, 2015; Published date: Jan 16, 2015

Copyright: ( 2015 Gokalp AS, et al. This is an open-access article distributed under the terms of the Creative Commons Attribution License, which permits unrestricted use, distribution, and reproduction in any medium, provided the original author and source are credited.

\section{Editorial}

The advances in neonatal intensive care in recent years have led to increases in the survival rates of babies that are born smaller. Prematurity is related to a chronic respiratory morbidity, which may persist until school age. However, this situation has caused extra problems including bronchopulmonary dysplasia (BPD). It was reported that preterm children, especially those with BPD, were faced with more frequent and severe respiratory symptoms compared to control groups of full-term newborns at school age and the adolescent period. The sequelae in childhood may be aggravated by atopy and passive smoking. Therefore, systematic follow-up programs, and lung function measurements are required in determining the severity of long-term lung dysfunction and in evaluating the efficacy of treatment.

Lung function can be evaluated by various methods such as conventional spirometry, respiratory inductance plethysmography and Impulse Oscillometry (IOS). However, most of the methods cannot be performed in small children due to problems with cooperation. The IOS method, which is a non-invasive method, is used to measure the impedance of input in the respiratory tract. More detailed information can be obtained with IOS compared to conventional spirometry. IOS is used to show the difference between central and peripheral components of airway resistance and to determine early pathological changes in the small airways. It detects the effects of treatment on various lung compartments. The values are recorded concurrently with each respiration during the measurement. It can be applied to small children because it does not require cooperation during the process.

There are many studies evaluating the long-term lung function of premature babies. These studies mostly involve the period of childhood and adolescence whereas the studies that cover late infancy and preschool age are limited. Most of the studies which investigate BPD patients' long-term pulmonary morbidity are usually performed during adolescence or young adulthood since performing pulmonary function tests requires cooperation of patients. There are few studies which cover infancy and/or preschool period.

Because preterm birth occurs at a sensitive period where lung maturation begins, pulmonary function tests performed in childhood depend on lung capacity at the time of birth as well as oxygen treatment, mechanical ventilation and a variety of medical treatments after birth. It is very difficult to compare the studies on this subject because many factors including atopy have been known to affect respiratory function. In fact, to investigate the effects of the premature birth, low birth weight and the severity of respiratory disease at the neonatal period on airway health during childhood independent from postnatal effects is impossible.

Conventional pulmonary function tests have difficulty in assessing lung function in preschool children due to required cooperation. In addition, coexisting neurological disorders of premature children make performing these tests more difficult. The oscillometric method enables the evaluation of various portions of the lungs during normal breathing. It is suitable to evaluate respiratory problems in preschool children because it requires minimal cooperation. One of our study showed that when premature babies are at the age of 3-4, they catch up their normal calendar age percentiles, but their lung function test results are not as successful as those of normal term babies. To understand how long the effects of being prematurely born and BPD continues, long-term studies with more cases are needed [1-7].

Therefore, Impulse Oscillometry (IOS) is seen a noninvasive and easily applicable method which can detect peripheral and central airway problems of the lungs in a sensitive way and will be used currently.

\section{References}

1. Baraldi E, Filippone M (2007) Chronic lung disease after premature birth. N Eng J Med 357: 1946.

2. Greenough A (2007) Late repiratory outcomes after preterm birth. Early Human Development 83: 785-788.

3. Narang I, Rosenthal M, Cremonesini D Silverman M, Bush A (2008) Longitudinal evaluation of airway function 21 years after preterm birth. Am J Respir Crit Care Med 178: 74-80.

4. Broström EB, Thunqvist P, Adenfelt G, Borling E, Katz-Salamon M (2010) Obstructive lung disease in children with mild to severe BPD. Respiratory Medicine 104: 362-370.

5. Hjalmarsonn O, Sandberg KL (2005) Lung function at term reflects severity of bronchopulmonary dysplasia. J Pediatr $146: 86-90$

6. Broughton S, Thomas MR, Marston L, Calvert SA, Marlow N, et al. (2007) Very prematurely born infants wheezing at follow-up: lung function and risk factors. Arch Dis Child 92: 776-780.

7. Fainardi V, Sapienza E, Teopompi E, Pisi G, Magnani Chetta A (2014) IOS in school-aged children born preterm: Is small for gestational age a risk factor for small airway dysfunction? European Respiratory Journal, 44: 1255. 